\title{
Characterization and modeling of mechanical behavior of single crystal titanium deformed by split-Hopkinson pressure bar
}

\author{
B. M. Morrow ${ }^{\mathrm{a}}$, R. A. Lebensohn ${ }^{\mathrm{a}}$, C. P. Trujillo ${ }^{\mathrm{a}}$, D. T. Martinez ${ }^{\mathrm{a}}$, F. L. Addessio ${ }^{\mathrm{a}}$, \\ C. A. Bronkhorst ${ }^{\mathrm{a}}$, T. Lookman ${ }^{\mathrm{a}}$, E. K. Cerreta $^{\mathrm{a}}$ \\ ${ }^{a}$ Los Alamos National Laboratory, P.O. Box 1663, MS G755, Los Alamos, NM 87545 USA
}

\begin{abstract}
Single crystal titanium samples were dynamically loaded using split-Hopkinson pressure bar (SHPB) and the resulting microstructures were examined. Characterization of the twins and dislocations present in the microstructure was conducted to understand the pathway for observed mechanical behavior. Electron backscatter diffraction (EBSD) was used to measure textures and quantify twinning. Microstructures were profusely twinned after loading, and twin variants and corresponding textures were different as a function of initial orientation. Focused ion beam (FIB) foils were created to analyze dislocation content using transmission electron microscopy (TEM). Large amounts of dislocations were present, indicating that plasticity was achieved through slip and twinning together. Viscoplastic Self-Consistent (VPSC) modeling was used to confirm the complex order of operations during deformation. The activation of different mechanisms was highly dependent upon crystal orientation. For [0001] and [1011]-oriented crystals, compressive twinning was observed, followed by secondary tensile twinning. Dislocations, though prevalent in the microstructure, contributed to final texture far less than twinning.
\end{abstract}

Keywords: microstructures, twinning, crystal plasticity, electron microscopy, Kolsky bar

\section{Introduction}

Materials properties in extreme environments are of great interest for structural applications. Of particular importance is the mechanical response of these materials in the complex dynamic loading environments experienced in automotive, aerospace, nuclear energy and biomedical applications. Hexagonal close-packed (hcp) metals, such as titanium, magnesium, and zirconium, and their alloys are important for their role as structural materials in these applications. As such, it is essential to understand the mechanical response under extreme conditions to prevent unexpected engineering failures of structural components.

Split-Hopkinson pressure bar (SHPB) is a common technique for studying mechanical properties of materials subjected to dynamic loading, where strain rates can exceed $10^{2} \mathrm{~s}^{-1}$

Email address: morrow@lanl.gov (B. M. Morrow)

Preprint submitted to International Journal of Plasticity

March 8, 2016 
(for a brief review, see (Kolsky, 1949; Gray, III, 2000; Ramesh, 2002)). The technique is well-suited for dynamic testing because it imparts a uniform, uniaxial load on a specimen. Much effort has been devoted to determining the predominant deformation mechanisms in hcp metals, notably titanium (Partridge, 1967; Christian and Mahajan, 1995; Nemat-Nasser et al., 1999; Meyers et al., 2001, 2002; Salem et al., 2003; Ungár et al., 2008; Moon et al., 2009; Xu et al., 2012), zirconium (Beyerlein and Tomé, 2008; McCabe et al., 2009; Morrow et al., 2013b; Niezgoda et al., 2013; Wang et al., 2013; Morrow et al., 2013a, 2014c), hafnium (Cerreta and Gray, III, 2004; Cerreta et al., 2007), and magnesium (Lou et al., 2007; El Kadiri et al., 2013; Morrow et al., 2014b,a, 2015) over a range of strain rates. Materials in this class tend to behave similarly, so for simplicity the current work focuses on titanium as a representative for ductile hcp materials. It is well known that both dislocation slip and twinning contribute significantly to the deformation behavior in polycrystalline hexagonal metals (Partridge, 1967; Christian and Mahajan, 1995), and that these mechanisms contribute differently depending on numerous conditions, including temperature, strain rate, grain size, polycrystalline texture, etc. The effects of strain rate, in particular, has proven challenging to predict. Several studies have observed the mechanical response of titanium under quasi-static (Nemat-Nasser et al., 1999; Salem et al., 2003; Zeng et al., 2009; Nixon et al., 2010) and dynamic loading (Meyers et al., 1994; Chichili et al., 1998; Nemat-Nasser et al., 1999; Cheng and Nemat-Nasser, 2000; Ramesh, 2002; Li et al., 2004; Huang et al., 2007; Xu et al., 2013; Sun et al., 2014). Existing dynamic mechanical test data, and, with it, understanding of active mechanisms in the dynamic loading regime is fairly limited. Because of this, few attempts have been made to predict the mechanical behavior of titanium under these extreme loading conditions. Additionally, most research to date has been performed on polycrystalline samples. Both twinning and slip, the mechanisms responsible for strength, occur predominately on the grain or single crystal length scale. It is therefore essential to understand plasticity in single crystals, which can then be used to inform polycrystalline strength models. Only a few works have dealt with the effect of crystallographic orientation on mechanical properties during quasi-static deformation in titanium (Battaini et al., 2007; Gong and Wilkinson, 2011). Generally, these studies are performed at strain rates below the dynamic regime, so the relative contributions and sensitivities of deformation mechanisms may differ from those observed at high rate.

The current work seeks to fill these gaps by studying titanium single crystals which have been deformed dynamically in compression using a SHPB technique. The resultant microstructure is characterized with respect to the basic deformation mechanisms: twinning and dislocation slip activity. Additionally, a visco-plastic self consistent (VPSC) model is used to simulate deformation in these single crystal samples during plastic deformation and determine the likely deformation path based on experimentally observed mechanisms.

\section{Material and Methods}

High-purity (99.99\%) titanium multi-crystalline materials were used for this study. The oxygen impurity content is expected to be less than $300 \mathrm{ppm}$. Electron backscatter diffraction (EBSD) was used to triage crystallographic orientations within the multi-crystals and 
identify large grains from which single crystal specimens would be machined. Cylindrical specimens in two different single crystal orientations, $2.5 \mathrm{~mm}$ in diameter by $2.5 \mathrm{~mm}$ tall, were machined from the larger multi-crystalline samples using electrical discharge machining (EDM). The single crystal specimens were machined such that a single orientation was parallel to the center compression axis of the cylinder in each case. The orientation of each smaller sample was verified using EBSD prior to mechanical testing. Top and bottom surfaces of each cylinder were scanned to ensure consistency through the thickness of each specimen. Fig. 1 shows EBSD maps for [0001] and [1011] specimens in the untested condition. Samples were deformed in uniaxial compression using a split-Hopkinson pressure bar (SHPB) at room temperature and to $25-30 \%$ strain. The compression direction corresponds to the normal axis of the pole figures shown in Fig. 1. Strain rates for each test were $\sim 4.45 \times 10^{3} \mathrm{~s}^{-1}$. After deformation, representative samples from each orientation were sectioned perpendicular to the loading direction and prepared using standard metallographic techniques. Fig. 2 shows light optical microscopy (LOM) of each sample after SHPB loading. Post-mortem EBSD was performed on an FEI Inspect SEM equipped with an EDAX EBSD system. An accelerating voltage of $20 \mathrm{kV}$ was used, with a step size of $0.35 \mu \mathrm{m}$. Post-mortem transmission electron microscopy (TEM) foils were prepared using an FEI Helios dual-beam focused ion beam (FIB) microscope. An FEI Tecnai with an accelerating voltage of $300 \mathrm{kV}$ was used for imaging.

\section{Results and Discussion}

Fig. 3 shows true stress as a function of true strain during dynamic loading for the two single crystal sample orientations obtained from the larger multi-crystalline specimen. The curves show very different mechanical behavior as a function of initial specimen orientation. To highlight the difference in mechanical response based on orientation, Fig. 4 shows stressstrain behavior for only two of the single crystals: one oriented for loading parallel to the [0001], and the other oriented parallel to [1011]. It is clear that the [0001] specimen exhibits a higher flow stress than the [1011] sample. This difference in behavior is typically associated with a difference in activity of the predominant deformation mechanism(s), in this case twinning versus dislocation slip. To confirm this hypothesis, these two specimens were selected for in depth microstructural characterization to correlate deformed structure to observed mechanical properties.

\subsection{Deformed Microstructure}

EBSD was used to measure orientation of the crystals after SHPB loading. Figs. 5 and 6 show inverse pole figure (IPF) maps of deformed [0001] and [1011] crystals, respectively. It is important to note that the scan data has been rotated to show orientations with respect to the loading direction to facilitate comparison with the initial, unstrained case. Both scans reveal a large amount of each crystal which had reoriented in a manner consistent with twinning. Additionally, material with a twin orientation relationship was observed within the primary twinned regions, indicating that second-generation (and potentially some latergeneration) twinning had occurred. Textures measured from EBSD, both before and after 
straining, are shown in Figs. 7 and 8. Changes in microstructure as a result of deformation become obvious by comparison. The initial, unstrained microstructures result in a single peak in the discrete texture plot shown in Figs. 7(a) and 8(a) (as the entire specimen is a single orientation). After deformation, several intense peaks are apparent in Figs. 7(b) and 8(b). Each strong peak corresponds to a specific orientation within the deformed microstructure. It is already known that twinning was prevalent in the deformed samples (see Figs. 5 and 6), and these peaks in Figs. 7(b) and 8(b) are consistent with twin orientations. To quantify the intensity or volume fraction of microstructure contributing to the reorientation peak, various regions of interest in each basal pole figure of deformed material were selected, shown in Fig. 9. The fraction of scan points in Figs. 5 and 6 with orientations corresponding to each region detailed in Fig. 9 were measured using TSL OIM Analysis software. Quantified results are shown in Tables 1 and 2 for the areas in Figs. 9(a) and 9(b), respectively.

The EBSD data quantify twinning, but are less well-suited to describe total dislocation content as a function of mechanical deformation. To access dislocation substructure, TEM was performed on each sample. Fig. 10 shows TEM micrographs for each orientation. Due to the very large amount of deformation in each specimen, a detailed characterization of individual dislocations was not possible. A very high dislocation density was observed in each sample, consistent with expectations for samples of this loading history. Simple tilting experiments were performed to check for dislocation invisibilities. Many of the dislocations maintained contrast while others became invisible under certain imaging conditions, indicating that there are multiple families of dislocations present in the samples, consistent with both $\langle a\rangle$-type and $\langle c+a\rangle$-type slip. Because each of the samples had experienced a large amount of strain, and therefore the microstructure had reoriented immensely from the initial, undeformed state, direct interpretation of the deformation behavior was difficult. Modeling was employed to discern the relative contributions of dislocations and twinning during deformation, and the order of the deformation evolution (in terms of slip and twin contributions) as a function of strain during mechanical testing.

\subsection{VPSC Modeling}

Visco-Plastic Self-Consistent (VPSC) modeling was chosen to reproduce the trends of experimentally observed textures and mechanical response of dynamically deformed Ti single crystals. This method has been successfully applied in the past to describe plastic deformation and texture evolution of polycrystalline hexagonal metals (Lebensohn and Tomé, 1993; Lebensohn and Canova, 1997; Tomé et al., 2001; Agnew et al., 2001; Proust et al., 2007; Nixon et al., 2010; Escobedo et al., 2012; Knezevic et al., 2013a). The model is wellsuited for this task because it is able to account for slip and twinning, strain-hardening, as well as macro-scale shear on the sample during loading. Given the initial and final textures, VPSC is able to determine the likely order of operative mechanisms during deformation, and the relative activity of each mechanism. Moreover, VPSC simulated hardening response can be compared with the experimental mechanical behavior to analyze, for example, the relative contributions of geometric hardening, which may become very relevant in twinning-dominated deformation, and strain hardening. 
Under VPSC, the constitutive behavior relating the stress, $\sigma$, and the strain rate, $\dot{\boldsymbol{\epsilon}}$, in a single crystal is given by:

$$
\dot{\boldsymbol{\epsilon}}=\sum_{s=1}^{N} \mathbf{m}^{s} \dot{\gamma}^{s}=\dot{\gamma}_{0} \sum_{s=1}^{N} \mathbf{m}^{s}\left(\frac{\left|\mathbf{m}^{s}: \boldsymbol{\sigma}\right|}{\tau^{s}}\right)^{n} \operatorname{sgn}\left(\mathbf{m}^{s}: \boldsymbol{\sigma}\right)
$$

where $\dot{\gamma}^{s}, \tau^{s}$, and $\boldsymbol{m}^{s}$ are, respectively, the shear rate, the critical resolved shear stress (CRSS) and the Schmid tensor, associated with slip or twinning system $s ; N$ is the total number of slip and twinning systems, $\dot{\gamma}_{0}$ is a normalization factor and $n$ is the stress exponent, inverse of the rate-sensitivity. Note that, in general, the CRSSs are a function of accumulated plastic strain in the crystal due to strain hardening. Here, we adopt the following extended Voce law (Tomé et al., 1984):

$$
\tau^{* s}=\tau_{0}^{s}+\left(\tau_{1}^{s}+\theta_{1}^{s} \Gamma\right)\left(1-\exp \left(-\Gamma\left|\frac{\theta_{0}^{s}}{\tau_{1}^{s}}\right|\right)\right)
$$

where $\Gamma=\sum_{s=1}^{N} \gamma^{s}$ is the total accumulated shear in the grain; $\tau_{0}^{s}, \tau_{1}^{s}, \theta_{0}^{s}$, and $\theta_{1}^{s}$ are the initial CRSS, the back-extrapolated CRSS, the initial hardening rate, and the asymptotic hardening rate, respectively, of slip or twinning system $s$. In addition, we allow for the possibility of self and latent hardening by defining coupling coefficients, $h^{s s^{\prime}}$, which empirically account for the obstacles that new dislocations or twins associated with system $s^{\prime}$ represent for the propagation of dislocations or twins on system $s$. The increase in the threshold stress after a time increment $\Delta t$ is calculated as:

$$
\Delta \tau^{s}=\frac{d \tau^{* s}}{d \Gamma} \sum_{s^{\prime}=1}^{N} h^{s s^{\prime}} \dot{\gamma}^{s^{\prime}} \Delta t
$$

Under VPSC, a set of single crystal grains represents the polycrystalline aggregate, and each of these grains may have different stress-strain rate responses, determined by its crystal orientation and its interaction with a homogenous equivalent medium whose effective properties are obtained as a weighted average over the aggregate of the local properties. Further details of the VPSC formulation can be found in (Lebensohn et al., 2007).

In this work, involving texture evolution by twinning of a single crystal, twinning reorientation was handled using the Predominant Twin Reorientation (PTR) scheme (Tomé et al., 1991), adapted to the case of an initial configuration consisting of a unique single crystal. In the case of polycrystals, PTR consists in using a MonteCarlo scheme to reorient entire grains with high twinning activity into the orientation of their most active twinning system, under the constraint that the total volume fraction of these reoriented grains has to coincide with the actual twinned volume fraction calculated throughout the aggregate. The twinned volume fraction associated with each grain is obtained as the ratio between the shears accommodated by twinning, as determined by the constitutive response, and the so-called "characteristic" twin shear that would result from the reorientation of the entire 
crystal. In this way, the overall twinned volume fraction is accounted for, while the number of orientations remains constant.

Mechanical behavior and texture evolution of the crystals compressed along the [0001] and [1011] directions were modeled by using 10,000 single crystal replicas, all with the same initial orientation. In the [0001] case, the c-axes of all replicas were initially parallel to the compression axis, while in the [1011] case, the [1011] directions were aligned with the compression axis. By application of the PTR scheme, if the constitutive response determines that, for example, $10 \%$ of the volume of the single crystal has twinned into one of the primary compressive twin variants, then 1,000 replicas will adopt the orientation of that variant. As soon as they are reoriented, these replicas will start accommodating shear by tensile twinning and some of them will eventually adopt the orientation of these tensile twins, reflecting the volume fraction of secondary twinning. In this way, the model can predict the volume fraction of every variant of primary compressive twins, secondary tensile twins and of the remaining original ("parent") single crystal.

All simulations were performed assuming that the Ti single crystal can deform plastically by $\{10 \overline{1} 0\}\langle 1 \overline{2} 10\rangle$ prismatic slip, and $\{10 \overline{1} 2\}\langle\overline{1} 011\rangle$ tensile and $\{11 \overline{2} 2\}\langle 11 \overline{2} \overline{3}\rangle$ compressive twinning. The prismatic slip mode consists of three different slip systems, while both the tensile and compressive twinning modes consist of six different variants, identified in what follows as: TTW1 through TTW6, and CTW1 through CTW6, respectively, given in Table 3. Each slip system and twin variant has a unique Schmid tensor entering in the constitutive equation (Eq. 1). For convenience, the value of $\dot{\gamma}_{0}$ was made to coincide with the applied strain rate: $4.45 \times 10^{3} \mathrm{~s}^{-1}$. The adopted value of the microscopic rate-sensitivity exponent was $n=6$, consistent with macroscopic experimental values (Chichili et al., 1998, 2004). The initial CRSSs, $\tau_{0}^{s}$, and the Voce hardening parameters, $\tau_{1}^{s}, \theta_{0}^{s}$, and $\theta_{1}^{s}$, associated with the slip and twinning modes are given in Table 4 . These were the values adopted in most cases presented below, except for one simulation performed under the assumption of no strain-hardening, i.e. $\tau_{1}^{s}, \theta_{0}^{s}, \theta_{1}^{s}=0$, to decouple and analyze the separate effect of geometric hardening. Concerning the adopted hardening matrix, two cases have been considered. Most examples below correspond to the assumption of no latent hardening, i.e. $h^{s s^{\prime}}=1, \forall s s^{\prime}$, except for one case of latent hardening only between compressive twinning variants, i.e. $h^{c t w, c t w}>1$, and $h^{s s^{\prime}}=1$ otherwise. In any event, given the lack of competition between different deformation modes, e.g. tension or compression along the c-axis of the parent orientation can be only accommodated by tensile or compressive twinning, respectively; the details of the adopted strain-hardening law have little effect on the predicted textures.

To gain insight on the mechanisms of texture and microstructure evolution of these Ti single crystals, predictions at an intermediate strain level (10\%) are reported, together with results at the maximum experimental strain level $(\sim 25 \%)$. Predictions at the final strain allow for direct comparison with experimental data, while results at the intermediate strain reveal information about the microstructural changes that lead to the final state, including the likely order in which deformation modes become dominant. EBSD data were used for calibration and validation of the VPSC predictions. Additionally, the LOM images of the deformed specimens (Fig. 2) were used to compare with the predicted macroscopic deformation of the samples. Like in the experiments, in the [0001] case symmetry determines 
zero macroscopic shear, while in the [1011] case a noticeable macroscopic shear is indeed predicted by the model.

In what follows, predicted textures are reported in the form of pole figures, in two ways: a) using individual dots corresponding to the poles associated with every active twin variant and, if still present, the parent single crystal, and b) using level lines representing volume fractions of the original single crystal reoriented into each twin variant and remaining in the initial orientation. While the latter predictions can be directly compared with the twinned volume fractions reported in Tables 1 and 2, in reference to the regions of the basal pole figure identified in Fig. 9, the representations of our simulated results should not be quantitatively compared with the pole figures reported in Figs. 7 and 8. In these experimental pole figures, intensity corresponds to multiples of random distribution and maxima associated with different twin variants are more spread, to the point that in some cases tensile and compressive twinning peaks merge into a single, widely spread maximum (see Fig. 7(b)). This can be due to lattice rotations associated with heterogeneous slip activity inside the twins, which are not captured by the VPSC model, and secondary slip modes other than prismatic slip (assumed in our simulations to be the only operative slip mode), which may also contribute to the orientation spread inside the twins.

Fig. 11 shows the basal (0001), prismatic $\{10 \overline{1} 0\}$ and pyramidal $\{10 \overline{1} 1\}$ pole figures corresponding to the initial Ti single crystal and from VPSC simulations after $10 \%$ and $25 \%$ compressive strain, with compression axis along the original [0001] crystal direction, represented in the center of the pole figures. The central spot in the basal pole figures at $0 \%$ and $10 \%$ strain corresponds to the parent crystal, and the six spots toward the rim, appearing after $10 \%$ strain, are the result of primary compressive twinning. Except for a rotation around the compression axis that is required to align the adopted initial orientation with the experimental data, these peaks are consistent with the regions of the basal pole figure labeled 2, 4, 6, 8, 10, and 12 in Fig. 9(a). The persistence of the central basal pole at $10 \%$ strain indicates that the model predicts that only a fraction of the parent crystal has twinned after $10 \%$ strain. In contrast, by the end of the simulation $(\epsilon=25 \%)$, no central basal intensity is left, indicating that the material is completely twinned. At $25 \%$ strain, the six primary compressive twin peaks persist and four secondary tensile twinning peaks have formed, which are consistent with four of the six odd numbered regions in Fig. 9a. Overall, after correcting for the aforementioned rotation around the compression axis, the predicted locations of the basal, prismatic and pyramidal poles after $25 \%$ strain are fairly consistent with the experimental textures after SHPB loading reported in Fig. 7(b).

Interestingly, the relative intensities of the strong texture peaks due to primary and subsequent secondary twinning appear to depend on the first variant that occurs in the microstructure. This indicates that, while for this very symmetric configuration all twin variants are a priori equally probable from an orientation point of view, certain variants are preferred after the first twin was formed. The final texture then follows from this initial twinning event. To illustrate this, Fig. 12 shows a basal pole figure, with the compression axis $x_{3}$ in the center and two mutually perpendicular axes $x_{1}^{\prime}$ and $x_{2}^{\prime}$ at right and top, respectively, showing the predicted volume fractions of the different primary compressive twin variants and the remaining original [0001] orientation, after $10 \%$ strain. Three pairs 
of opposed twin variants have accumulated different volume fractions of material, ranging from $7 \%$ to $20 \%$. Furthermore, Fig. 13 shows the corresponding evolved textures after $25 \%$ strain. Table 5 shows the predicted volume fractions of the different primary and secondary twin variants. The arrows in Fig. 13(a) indicate in which primary twin variant secondary twins were originated. Note that in order to directly compare these predictions with the experimental results (Fig. 9(a)), axes $x_{1}$ and $x_{2}$ (corresponding to a rotation around compression axis $x_{3}$ ) were identified to give the best match between the predicted and measured intensities for regions 1 through 13. Fig. 13(b) shows the predicted (in black) and measured (in color) volume fractions of the different labeled regions/twin variants. Measured volume fractions in green indicate reasonable agreement with the predictions, while red numbers indicate poor matching.

Next, the case of the Ti single crystal compressed along the [1011] direction is analyzed. Fig. 14 shows the basal (0001), prismatic $\{10 \overline{1} 0\}$ and pyramidal $\{10 \overline{1} 1\}$ pole figures corresponding to the initial configuration and from VPSC simulations after 10\% and 25\% compression along the original [1011] crystal direction. Note the match between the initial single crystal orientation and the experimental pole figure, Fig. 8(a). The single spot in the initial basal pole figure remains after $25 \%$ strain, indicating that, unlike the previous case and consistent with the experimental evidence, the initial single crystal is not completely consumed in the recovered sample. The two basal poles in the upper quadrants observed at both $10 \%$ and $25 \%$ strain correspond to primary compressive twinning, while the basal pole observed near the compression axis at $25 \%$ strain corresponds to a single variant of secondary tensile twinning. More details of the texture evolution can be discerned in the basal pole projections of Figs. 15 and 16, in which level lines indicate the predicted volume fractions of each twin variant and the parent single crystal, for $10 \%$ and $25 \%$ strain, respectively. At 10\% strain (Fig. 15) the model predicts that a 35\% volume fraction of the specimen has twinned into two compressive twinning variants having basal poles almost perpendicular to the compression axis, with one variant accommodating roughly two times more deformation than the other. At 25\% strain, Fig. 16 shows that inside the most active compressive twinning variant (CTW2), one tensile twinning variant (TTW2) was formed, with its (0001) pole direction re-aligned with the compression direction. Table 6 shows the predicted volume fractions of the different primary and secondary twin variants. It is evident that the model overestimates the total twinned volume $(\sim 75 \%)$ compared with the measured one ( $\sim 55 \%)$. Fig. 16(a) also shows regions 1-2 and 5-8 of the experimental basal pole figure after SHPB loading reported in Fig. 9(b). Fig. 16(b) shows the volume fractions predicted (in black) and measured (in green/red for good/poor agreement with the predictions) of the different labeled regions/twin variants. Evidently, the predicted parent, TTW2, CTW3 and CTW2 basal maxima are close to the four most intense experimental maxima, labeled 1,2,6, and 8, respectively, except for an upward displacement that would need to be experienced by the predicted peaks for an almost ideal matching. It is worth noting that the model predicts a macroscopic positive shear along direction $x_{2}$ normal to direction $x_{3}$ of $\sim 0.25$ after $25 \%$ compressive strain, which is consistent with the shape of the recovered sample. This shear also determines lattice rotations (of $\sim 6$ and $\sim 14$ degrees after $10 \%$ and $25 \%$ strain, respectively) that do displace the basal pole maxima upwards, but not enough 
to make them coincide with the experimental peaks. This quantitative disagreement may be due to either an underestimation of the actual shear by the model, or uncertainties in the alignment during EBSD characterization of the sheared recovered sample. In any event, in this case the model provides a plausible explanation of the interplay between observed texture and the twinning-dominated microstructure evolution.

The above observations on the role of twinning in microstructure evolution and texture development are summarized in Fig. 17, which shows the relative activity of the slip and twinning modes, together with the evolution of the compressive and tensile twinning volume fractions for both initial crystal orientations. It should be noted that Fig. 17 depicts the VPSC model predictions, which compare favorably (at least in end-point behavior) with the experimental observations. In the case of the [0001] crystal, given that prismatic slip is unable to accommodate strain along the $\langle c\rangle$-axis, compressive twinning clearly dominates the early stage of deformation, determining that $100 \%$ of the parent grain has twinned after $20 \%$ strain. On the other hand, the dominant deformation mode in the [1011] crystal is the soft (and well-oriented, in this case) prismatic slip. With a smaller contribution, primary compressive twinning is not completely exhausted after $25 \%$ strain. Due to the unfavorable orientations of both parent crystals for tensile twinning activation, this mode requires the prior formation of primary compressive twins. Therefore, this secondary twinning mechanism starts contributing to plastic deformation after $\sim 5-10 \%$ strain. While some of the deformation modes are similar to those expected from polycrystalline behavior (i.e. Xu et al. 2013) the activities and hardening behavior is expected to be different, as single crystalline samples will involve only intragranular mechanisms, while polycrystalline samples will have intergranular effects also.

Finally, we present the effect of different strain hardening assumptions on the predicted stress-strain response. It is important to reiterate that the adoption of different hardening laws has a minor effect on the texture evolution discussed previously, due to a very strong dependence of the favorability of the different deformation modes with crystal orientation, irrespective of the precise value of their critical stresses. In the first case, the strain hardening was set to zero to discern the effect of geometric hardening only. The resulting stressstrain curves are shown in Fig. 18(a). The [0001] case has a slightly higher initial yield stress. For both parent crystal orientations, the initial flat behavior (up to $\sim 5 \%$ strain) is due to a known issue of the PTR scheme (Tomé et al., 1991), i.e. a lagging effect that requires a few deformation steps to equalize the volume fraction actually reoriented into the twin orientation with the effective twinned volume fraction determined by the constitutive behavior. Once the latter is achieved, both configurations show a strong hardening associated with the rapid increase of the volume fraction of compressive twinning, a mechanism that flips material into much harder orientations with respect to the applied compressive loading. Then, when the activity of softer tensile twinning increases, both stress-strain curves become flat. In both cases, geometric hardening can account for roughly a $50 \%$ increase in flow stress after $25 \%$ strain. This is evidently not enough to explain the measured $\sim 4$-fold increase, reported in Fig. 3. To approach this order of magnitude and attempt a plausible explanation for the differences between the two experimental curves, Fig. 18(b) shows the cases with strain hardening parameters given in Table 4, corresponding to two different assumptions 
for the hardening matrix $h^{s s^{\prime}}$ (Eq. 3): i) no latent hardening, i.e. $h^{s s^{\prime}}=1$, and and ii) strong latent hardening, only between compressive twinning variants, i.e. $h^{c t w, c t w^{\prime}}=8$ and $h^{s s^{\prime}}=1$ otherwise. The trend observed in Fig. 3, wherein the [0001] crystal hardens faster than the [1011] crystal, can be achieved by the imposed latent hardening on compressive twinning interactions. In the [0001] case, symmetry determines that all six compressive twinning variants are activated, which makes CTW-CTW' latent hardening very effective to increase the critical stresses of compressive twinning, and, concomitantly, the macroscopic stress. In the [1011] case, compressive twinning activity is lower and only two variants are active, making the adopted latent hardening less effective in increasing the effective stress response. On the other hand, the clear change in hardening rate of the [0001] stress-strain curve, contrasting with the fairly constant rate of the [1011] curve, observed in Fig. 4, cannot be obtained with the relatively simple Voce hardening law given by Eqs. (2-3). More sophisticated laws, including explicit consideration of the dislocation densities and slip-twin interactions (e.g. (Knezevic et al., 2013b)) should be adopted and identified to capture the observed trend. The consideration and calibration of such improved hardening description will be reported in the future.

\section{Conclusions}

Titanium single crystals were deformed in compression by SHPB loading. Crystals in the [0001] and [1011] orientations were chosen for detailed analysis. Each orientation showed very high dislocation density, and high twin fraction following deformation, indicating that both mechanisms play an important role in the observed mechanical behavior. The relative contributions of each mechanism was markedly different for the two orientations. Total twin fractions of $99 \%$ and $66 \%$ for [0001] and [1011] orientations, respectively, were measured using EBSD. Second generation twins were prevalent, especially in the [0001] orientation. Such a large volume of transformed material made direct interpretation difficult, so VPSC modeling was used to determine the deformation evolution during dynamic loading. VPSC indicated that, while dislocation activity was important, massive reorientations of the microstructure occurred due to twinning. For the [0001] case, the initial, undeformed crystal deformed by first forming several variants of compression twins. At later stages of strain, the first-generation twins often underwent second-generation tensile twinning. The preferred variants of twins formed during deformation seem to be geometrically dependent on the firstgeneration twin variant that nucleates first. Third-generation twinning was also observed, though did not contribute significantly to the final texture. For the [1011] case, due to the orientation of the parent crystal, only two variants of compression twin were predominant. At higher strains, second-generation tensile twinning formed within the more active variant. In general, VPSC simulations showed good agreement with experimental observations on microstructure and texture evolution. Additionally, no macroscale shear was observed in the [0001] crystal, but shear was seen in the [1011] case. This was also observed in simulations. Finally, VPSC modeling was used to reproduce the hardening behavior of the single crystals as a function of strain, yielding good qualitative agreement with experimental mechanical data, in terms of the relative response of both single crystal orientations. Simulation of more 
detailed characteristics of the stress-strain response requires adoption of more sophisticated hardening laws at the single crystal level.

\section{Acknowledgements}

Los Alamos National Laboratory is operated by LANS, LLC, for the NNSA of the U.S. Department of Energy under contract DE-AC52-06NA25396. Campaign 2 programs supported this work. The authors are grateful to Program Managers Rick Martineau, Russ Olson, and Sherri Bingert. LA-UR-15-26741.

\section{References}

Agnew, S.R., Yoo, M.H., Tomé, C.N., 2001. Application of texture simulation to understanding mechanical behavior of $\mathrm{Mg}$ and solid solution alloys containing Li or Y. Acta Materialia 49, 4277-4289.

Battaini, M., Pereloma, E.V., Davies, C.H.J., 2007. Orientation effect on mechanical properties of commercially pure titanium at room temperature. Metallurgical and Materials Transactions A 38, $276-285$.

Beyerlein, I.J., Tomé, C.N., 2008. A dislocation-based constitutive law for pure Zr including temperature effects. International Journal of Plasticity 24, 867-895.

Cerreta, E., Yablinsky, C.A., Gray, III, G.T., Vogel, S.C., Brown, D.W., 2007. The influence of grain size and texture on the mechanical response of high purity hafnium. Materials Science and Engineering A $456,243-251$.

Cerreta, E.K., Gray, III, G.T., 2004. The influence of texture, strain rate, temperature, and chemistry on the mechanical behavior of hafnium. Metallurgical and Materials Transactions A 35, 1999-2011.

Cheng, J., Nemat-Nasser, S., 2000. A model for experimentally-observed high-strain-rate dynamic strain aging in titanium. Acta Materialia 48, 3131-3144.

Chichili, D.R., Ramesh, K.T., Hemker, K.J., 1998. The high-strain-rate response of alpha-titanium: experiments, deformation mechanisms and modeling. Acta Materialia 46, 1025-1043.

Chichili, D.R., Ramesh, K.T., Hemker, K.J., 2004. Adiabatic shear localization in $\alpha$-titanium: experiments, modeling and microstructural evolution. Journal of the Mechanics and Physics of Solids 52, 1889-1909.

Christian, J.W., Mahajan, S., 1995. Deformation twinning. Progress in Materials Science 39, 1-157.

El Kadiri, H., Baird, J.C., Kapil, J., Oppedal, A.L., Cherkaoui, M., Vogel, S.C., 2013. Flow asymmetry and nucleation stresses of twinning and non-basal slip in magnesium. International Journal of Plasticity 44, 111-120.

Escobedo, J.P., Cerreta, E.K., Trujillo, C.P., Martinez, D.T., Lebensohn, R.A., Gray, III, G.T., 2012. Influence of texture and test velocity on the dynamic, high-strain, tensile behavior of zirconium. Acta Materialia 60, 4379-4392.

Gong, J., Wilkinson, A.J., 2011. Micro-cantilever testing of <a>prismatic slip in commercially pure Ti. Philosophical Magazine 91, 1137-1149.

Gray, III, G.T., 2000. Classic split-hopkinson pressure bar testing. ASM Handbook , 462-476.

Huang, W., Zan, X., Nie, X., Gong, M., Wang, Y., Xia, Y., 2007. Experimental study on the dynamic tensile behavior of a poly-crystal pure titanium at elevated temperatures. Materials Science and Engineering: A 443, 33-41.

Knezevic, M., Lebensohn, R.A., Cazacu, O., Proust, G., Vogel, S., Nixon, M.E., 2013a. Modeling bending of alpha-titanium with embedded polycrystal plasticity in implicit finite elements. Materials Science \& Engineering: A 564, 116-126.

Knezevic, M., McCabe, R.J., Tomé, C.N., Lebensohn, R.A., Chen, S.R., Cady, C.M., Gray, III, G.T., Mihaila, B., 2013b. Modeling mechanical response and texture evolution of $\alpha$-uranium as a function of strain rate and temperature using polycrystal plasticity. International Journal of Plasticity 43, 70-84.

Kolsky, H., 1949. An investigation of the mechanical properties of materials at very high rates of loading. Proceedings of the Physical Society. Section B 62, 676. 
Lebensohn, R.A., Canova, G.R., 1997. A self-consistent approach for modelling texture development of two-phase polycrystals: application to titanium alloys. Acta Materialia 45, 3687-3694.

Lebensohn, R.A., Tomé, C.N., 1993. A self-consistent anisotropic approach for the simulation of plastic deformation and texture development of polycrystals: Application to zirconium alloys. Acta Metallurgica et Materialia 41, 2611-2624.

Lebensohn, R.A., Tomé, C.N., Ponte Castañeda, P., 2007. Self-consistent modelling of the mechanical behaviour of viscoplastic polycrystals incorporating intragranular field fluctuations. Philosophical Magazine 87, 4287-4322.

Li, Q., Xu, Y.B., Bassim, M.N., 2004. Dynamic mechanical behavior of pure titanium. Journal of Materials Processing Technology 155-156, 1889-1892. Proceedings of the International Conference on Advances in Materials and Processing Technologies: Part 2.

Lou, X., Li, M., Boger, R., Agnew, S., Wagoner, R., 2007. Hardening evolution of az31b mg sheet. International Journal of Plasticity 23, 44-86.

McCabe, R.J., Proust, G., Cerreta, E.K., Misra, A., 2009. Quantitative analysis of deformation twinning in zirconium. International Journal of Plasticity 25, 454-472.

Meyers, M.A., Benson, D.J., Vöhringer, O., Kad, B.K., Xue, Q., Fu, H.H., 2002. Constitutive description of dynamic deformation: physically-based mechanisms. Materials Science and Engineering A 322, $194-216$.

Meyers, M.A., Subhash, G., Kad, B.K., Prasad, L., 1994. Evolution of microstructure and shear-band formation in $\alpha$-hcp titanium. Mechanics of Materials 17, 175-193.

Meyers, M.A., Vöhringer, O., Lubarda, V.A., 2001. The onset of twinning in metals: a constitutive description. Acta Materialia 49, 4025-4039.

Moon, J.H., Karthikeyan, S., Morrow, B.M., Fox, S.P., Mills, M.J., 2009. High-temperature creep behavior and microstructure analysis of binary $\mathrm{Ti}-6 \mathrm{Al}$ alloys with trace amounts of Ni. Materials Science and Engineering A 510-511, 35-41.

Morrow, B.M., Cerreta, E.K., McCabe, R.J., Tomé, C.N., 2014a. Toward understanding twin-twin interactions in hcp metals: Utilizing multiscale techniques to characterize deformation mechanisms in magnesium. Materials Science \& Engineering: A 613, 365-371.

Morrow, B.M., Cerreta, E.K., McCabe, R.J., Tomé, C.N., 2015. Transmission electron microscope in situ straining technique to directly observe defects and interfaces during deformation in magnesium. JOM 67, $1721-1728$.

Morrow, B.M., Kozar, R.W., Anderson, K.R., Mills, M.J., 2013a. An examination of the use of the Modified Jogged-Screw model for predicting creep behavior in Zircaloy-4. Acta Materialia 61, 4452-4460.

Morrow, B.M., McCabe, R.J., Cerreta, E.K., Tomé, C.N., 2013b. Variability in ebsd statistics for textured zirconium. Materials Science and Engineering: A 574, 157-162.

Morrow, B.M., McCabe, R.J., Cerreta, E.K., Tomé, C.N., 2014b. In-situ TEM observation of twinning and detwinning during cyclic loading in Mg. Metallurgical and Materials Transactions A 45, 36-40.

Morrow, B.M., McCabe, R.J., Cerreta, E.K., Tomé, C.N., 2014c. Observations of the atomic structure of tensile and compressive twin boundaries and twin-twin interactions in zirconium. Metallurgical and Materials Transactions A 45, 5891-5897.

Nemat-Nasser, S., Guo, W.G., Cheng, J.Y., 1999. Mechanical properties and deformation mechanisms of a commercially pure titanium. Acta Materialia 47, 3705-3720.

Niezgoda, S.R., Kanjarla, A.K., Beyerlein, I.J., Tomé, C.N., 2013. Stochastic modeling of twin nucleation in polycrystals: An application in hexagonal close-packed metals. International Journal of Plasticity , - .

Nixon, M.E., Cazacu, O., Lebensohn, R.A., 2010. Anisotropic response of high-purity $\alpha$-titanium: Experimental characterization and constitutive modeling. International Journal of Plasticity 26, 516-532.

Partridge, P.G., 1967. The crystallography and deformation modes of hexagonal close-packed metals. Metallurgical Reviews 12, 169-194.

Proust, G., Tomé, C.N., Kaschner, G.C., 2007. Modeling texture, twinning and hardening evolution during deformation of hexagonal materials. Acta Materialia 55, 2137-2148.

Ramesh, K.T., 2002. Effects of high rates of loading on the deformation behavior and failure mechanisms of hexagonal close-packed metals and alloys. Metallurgical and Materials Transactions A 33, 927-935. 
Salem, A.A., Kalidindi, S.R., Doherty, R.D., 2003. Strain hardening of titanium: role of deformation twinning. Acta Materialia 51, 4225-4237.

Sun, J.L., Trimby, P.W., Yan, F.K., Liao, X.Z., Tao, N.R., Wang, J.T., 2014. Shear banding in commercial pure titanium deformed by dynamic compression. Acta Materialia 79, 47-58.

Tomé, C.N., Canova, G.R., Kocks, U.F., Christodoulou, N., Jonas, J.J., 1984. The relation between macroscopic and microscopic strain hardening in FCC polycrystals. Acta Metallurgica 32, 1637-1653.

Tomé, C.N., Lebensohn, R.A., Kocks, U.F., 1991. A model for texture development dominated by deformation twinning: Application to zirconium alloys. Acta Metallurgica et Materialia 39, 2667-2680.

Tomé, C.N., Maudlin, P.J., Lebensohn, R.A., Kaschner, G.C., 2001. Mechanical response of zirconium-I. Derivation of a polycrystal constitutive law and finite element analysis. Acta Materialia 49, 3085-3096.

Ungár, T., Glavicic, M.G., Balogh, L., Nyilas, K., Salem, A.A., Ribárik, G., Semiatin, S.L., 2008. The use of $\mathrm{x}$-ray diffraction to determine slip and twinning activity in commercial-purity $(\mathrm{CP})$ titanium. Materials Science and Engineering: A 493, 79-85. Mechanical Behavior of Nanostructured Materials, a Symposium Held in Honor of Carl Koch at the \{TMS \} Annual Meeting 2007, Orlando, Florida.

Wang, H., Wu, P.D., Wang, J., Tomé, C.N., 2013. A crystal plasticity model for hexagonal close packed (hcp) crystals including twinning and de-twinning mechanisms. International Journal of Plasticity 49, $36-52$.

Xu, F., Zhang, X., Ni, H., Cheng, Y., Zhu, Y., Liu, Q., 2013. Effect of twinning on microstructure and texture evolutions of pure ti during dynamic plastic deformation. Materials Science and Engineering: A $564,22-33$.

Xu, F., Zhang, X., Ni, H., Liu, Q., 2012. $\{11 \overline{2} 4\}$ deformation twinning in pure Ti during dynamic plastic deformation. Materials Science and Engineering: A 541, 190-195.

Zeng, Z., Jonsson, S., Roven, H.J., 2009. The effects of deformation conditions on microstructure and texture of commercially pure ti. Acta Materialia 57, 5822-5833.

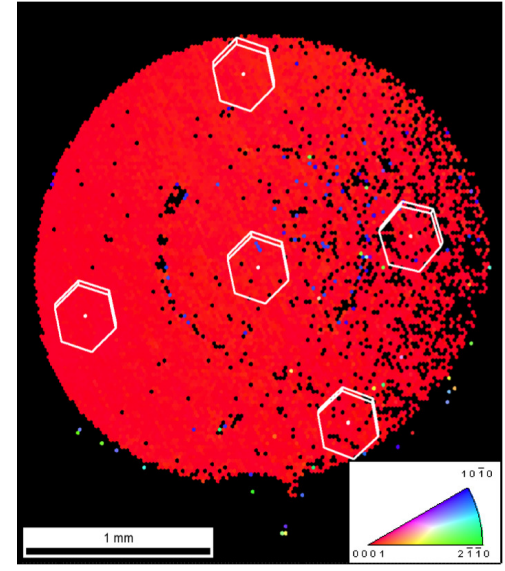

(a) $[0001]$

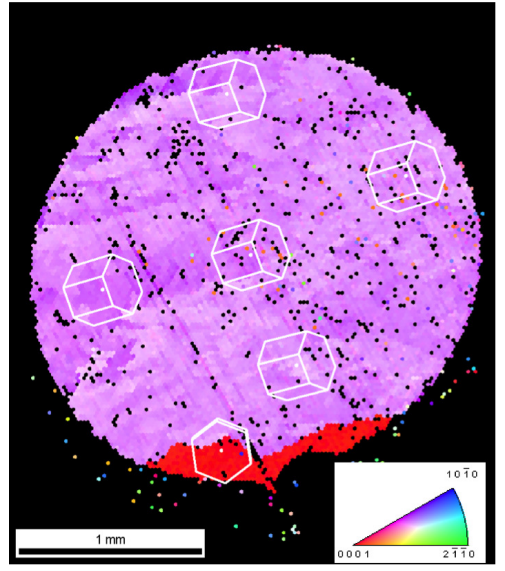

(b) $[10 \overline{1} 1]$

Figure 1: EBSD of compression surface of (a) [0001], and (b) [10111] specimens before loading. 

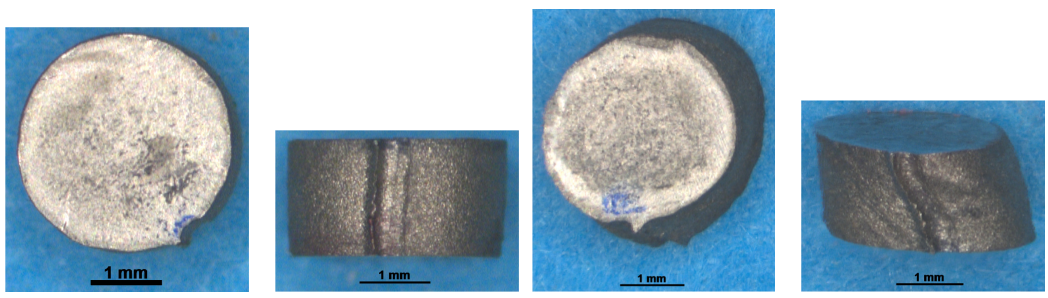

(a) Impact Sur- (b) Side View,(c) Impact Sur- (d) Side View, face, [0001] crys- [0001] crystal face, [101̄1] crys- [10̄11] crystal tal tal

Figure 2: Light optical microscopy of (a)-(b) [0001], and (c)-(d) [1011] specimens after SHPB loading.

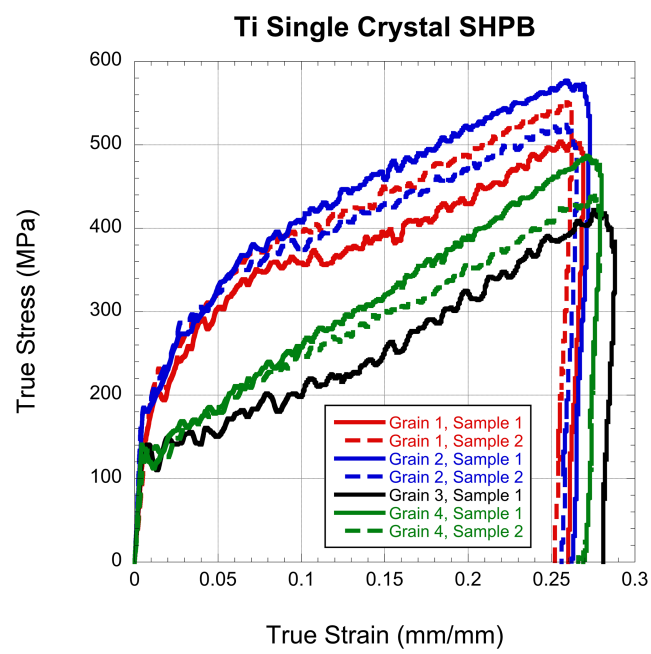

Figure 3: Stress-strain curves for Ti single crystal samples loaded using SHPB.



Figure 4: Stress-strain curves for two representative orientations of Ti single crystal, loaded using SHPB. 


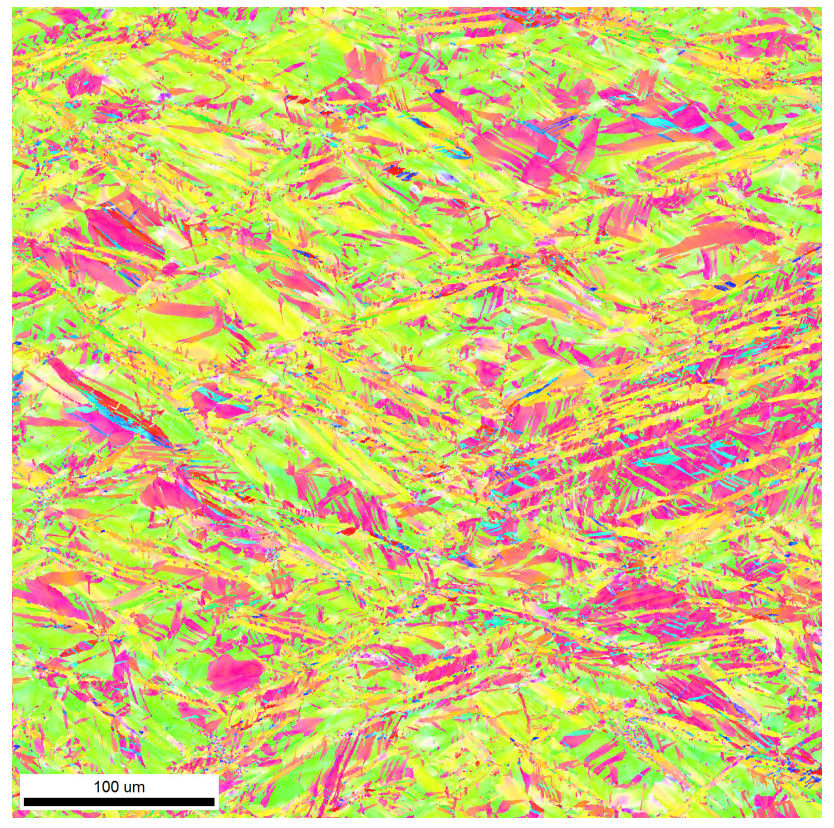

(a)

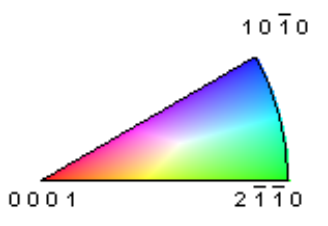

(b)

Figure 5: Inverse Pole Figure from EBSD scan of [0001] oriented Ti single crystal after loading with SHPB.

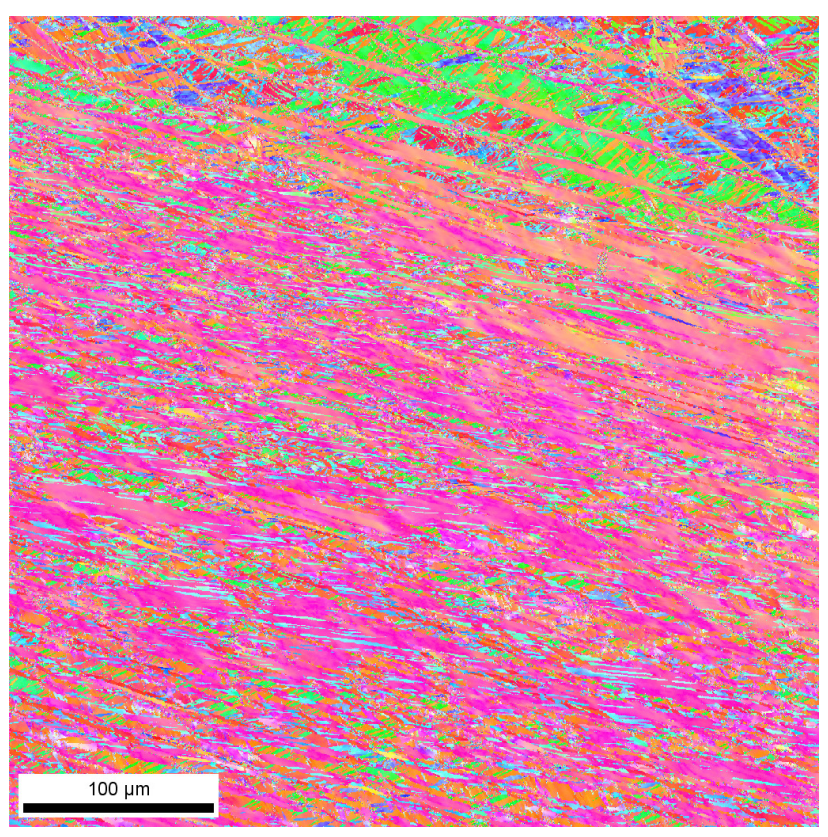

(a)

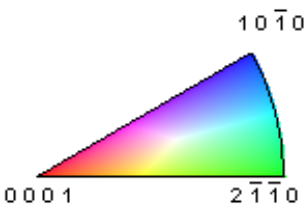

(b)

Figure 6: Inverse Pole Figure from EBSD scan of [10111] oriented Ti single crystal after loading with SHPB. 



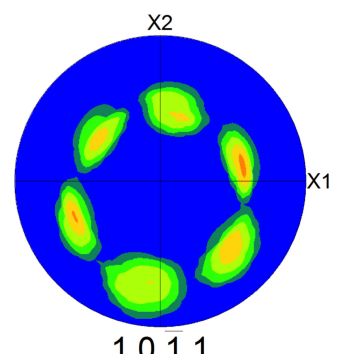

(a) Unstrained

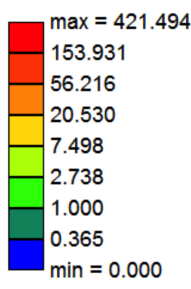



(b) After SHPB Loading

Figure 7: Texture plots from EBSD from [0001] crystal in (a) unstrained sample, and (b) sample deformed to $\sim 25 \%$ strain.
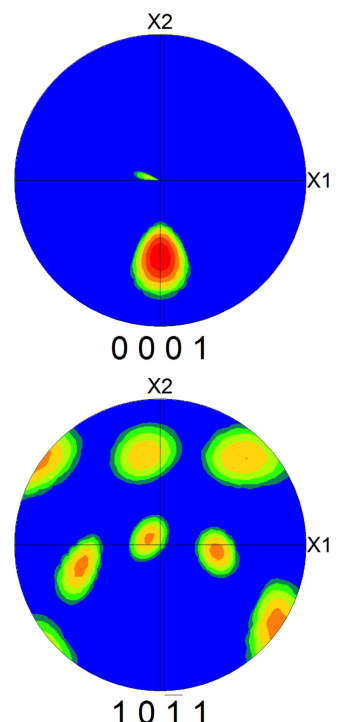

(a) Unstrained
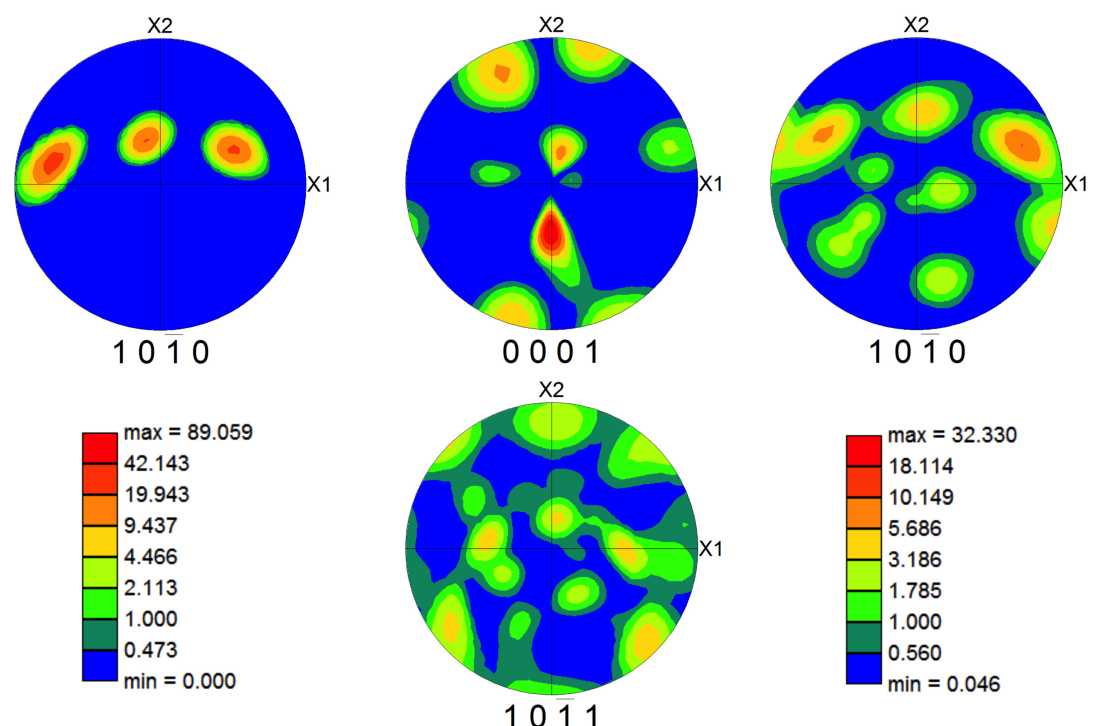

(b) After SHPB Loading

Figure 8: Texture plots from EBSD from [10̄11] crystal in (a) unstrained sample, and (b) sample deformed to $\sim 25 \%$ strain. 


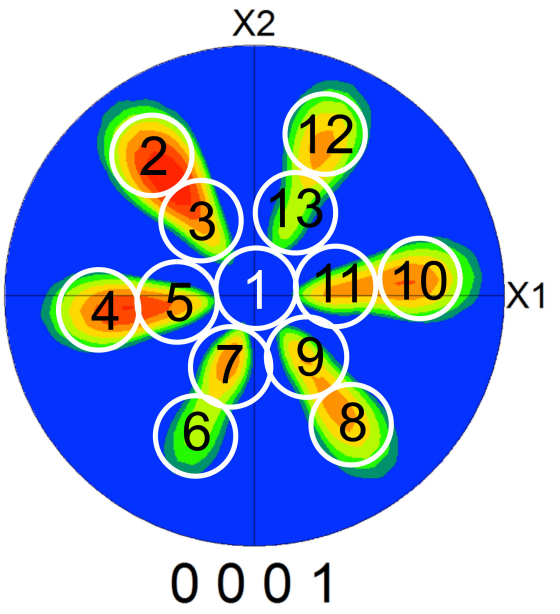

(a) [0001] crystal

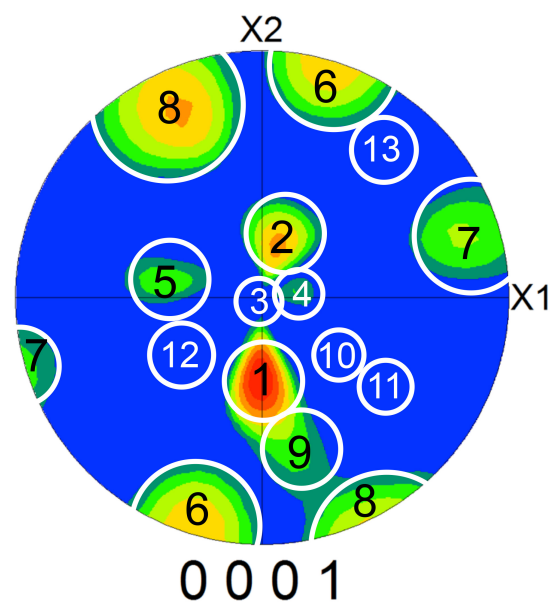

(b) $[10 \overline{1} 1]$ crystal

Figure 9: Texture plots from EBSD from (a) [0001] crystal, and (b) [1011] crystal, with locations for texture intensity analysis in Tables 1 and 2 shown.

Table 1: Fraction of EBSD scan points with orientation within each numbered region of interest in texture plot (Fig. 9(a)).

\begin{tabular}{|c|c|}
\hline Location & Scan Fraction \\
\hline 1 & 0.010 \\
\hline 2 & 0.155 \\
\hline 3 & 0.107 \\
\hline 4 & 0.108 \\
\hline 5 & 0.102 \\
\hline 6 & 0.030 \\
\hline 7 & 0.099 \\
\hline 8 & 0.067 \\
\hline 9 & 0.064 \\
\hline 10 & 0.083 \\
\hline 11 & 0.075 \\
\hline 12 & 0.069 \\
\hline 13 & 0.041 \\
\hline
\end{tabular}


Table 2: Fraction of EBSD scan points with orientation within each numbered region of interest in texture plot (Fig. 9(b)).

\begin{tabular}{|c|c|}
\hline Location & Scan Fraction \\
\hline 1 & 0.440 \\
\hline 2 & 0.196 \\
\hline 3 & 0.025 \\
\hline 4 & 0.036 \\
\hline 5 & 0.035 \\
\hline 6 & 0.068 \\
\hline 7 & 0.031 \\
\hline 8 & 0.110 \\
\hline 9 & 0.022 \\
\hline 10 & 0.009 \\
\hline 11 & 0.005 \\
\hline 12 & 0.002 \\
\hline 13 & 0.002 \\
\hline
\end{tabular}

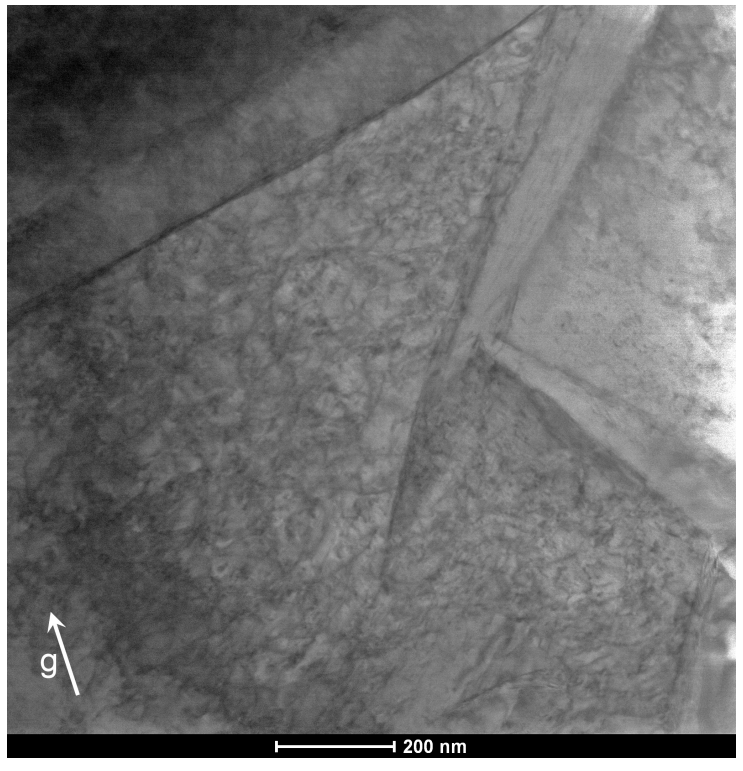

(a)

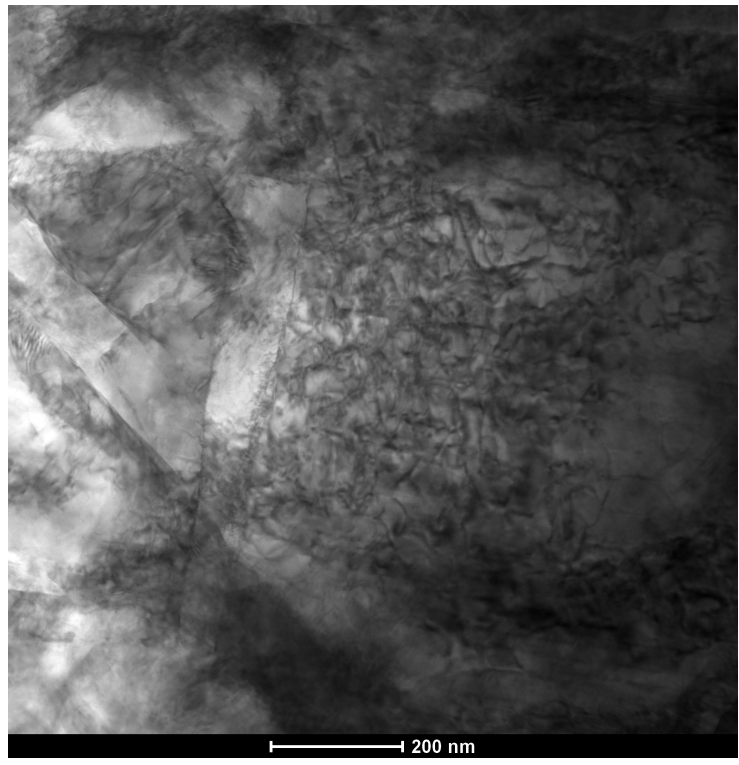

(b)


high dislocation densities. 
Table 3: Twin variantes of the tensile and compressive twinning modes.

\begin{tabular}{|c|c|c|}
\hline Twin Variant & Twin Plane & Twin Direction \\
\hline TTW1 & $(10 \overline{1} 2)$ & {$[\overline{1} 011]$} \\
\hline TTW2 & $(01 \overline{1} 2)$ & {$[0 \overline{1} 11]$} \\
\hline TTW3 & $(\overline{1} 102)$ & {$[1 \overline{1} 01]$} \\
\hline TTW4 & $(\overline{1} 012)$ & {$[10 \overline{1} 1]$} \\
\hline TTW5 & $(0 \overline{1} 12)$ & {$[01 \overline{1} 1]$} \\
\hline TTW6 & $(1 \overline{1} 02)$ & {$[\overline{1} 101]$} \\
\hline CTW1 & $(2 \overline{1} \overline{1} 2)$ & {$[2 \overline{1} \overline{1} 3]$} \\
\hline CTW2 & $(1 \overline{1} 2)$ & {$[11 \overline{2} \overline{3}]$} \\
\hline CTW3 & $(\overline{1} 2 \overline{1} 2)$ & {$[\overline{1} 2 \overline{1} \overline{3}]$} \\
\hline CTW4 & $(\overline{2} 112)$ & {$[\overline{2} 11 \overline{3}]$} \\
\hline CTW5 & $(\overline{1} \overline{1} 22)$ & {$[\overline{1} \overline{1} 2 \overline{3}]$} \\
\hline CTW6 & $(1 \overline{2} 12)$ & {$[1 \overline{2} 1 \overline{3}]$} \\
\hline
\end{tabular}

Table 4: Adopted initial CRSSs and Voce hardening parameters for the slip and twinning modes.

\begin{tabular}{|c|c|c|c|c|}
\hline Mode & $\tau_{0}^{s}[M P a]$ & $\tau_{1}^{s}[M P a]$ & $\theta_{0}^{s}[M P a]$ & $\theta_{1}^{s}[M P a]$ \\
\hline Prismatic Slip & 10 & 0 & 15 & 15 \\
\hline Tensile Twinning & 25 & 0 & 37.5 & 37.5 \\
\hline Compressive Twinning & 50 & 0 & 75 & 75 \\
\hline
\end{tabular}



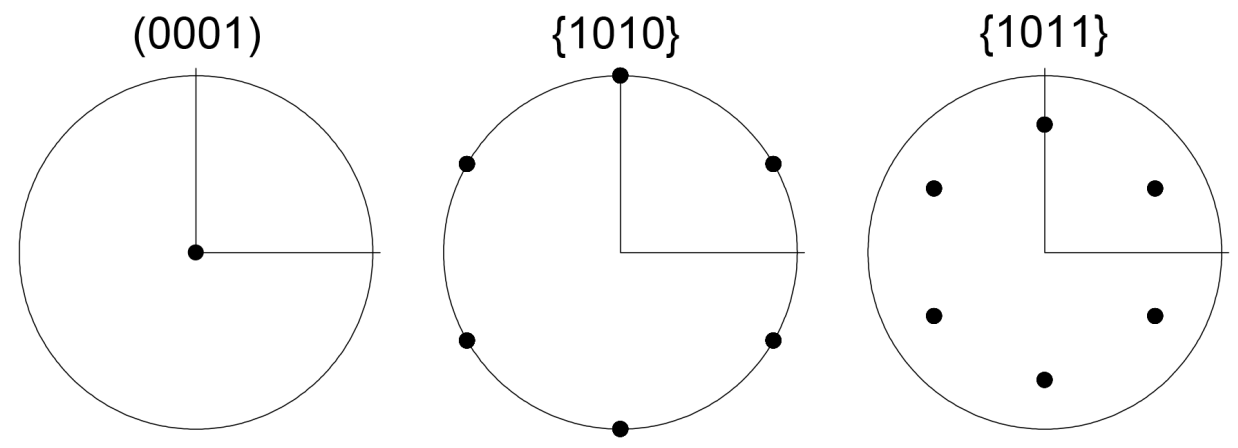

\section{initial}
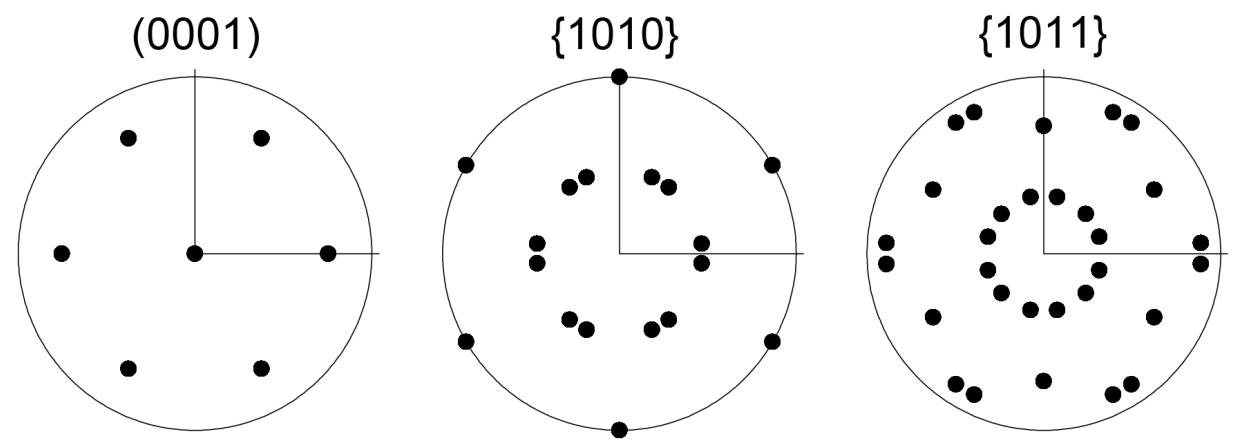

$10 \%$
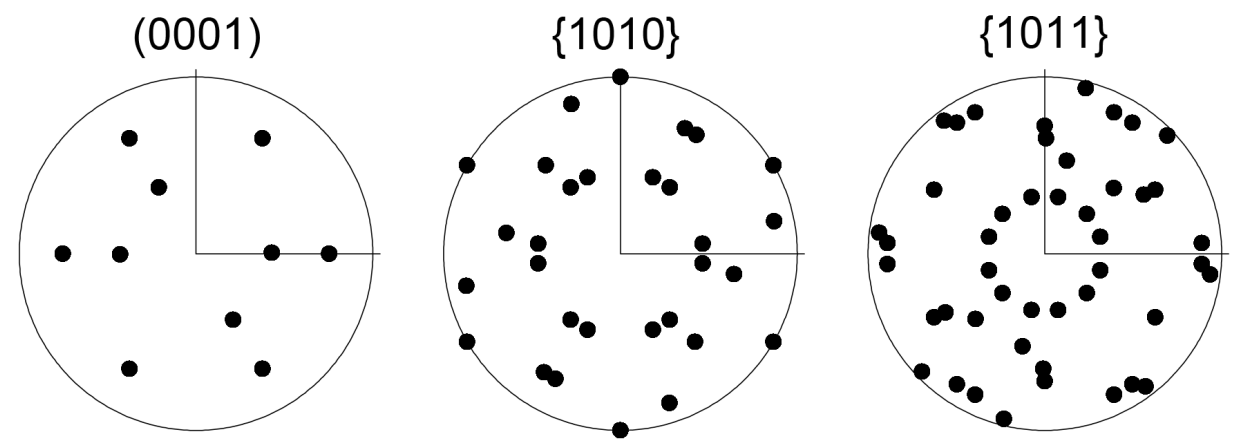

$25 \%$

Figure 11: Basal (0001), prismatic $\{10 \overline{1} 0\}$, and pyramidal $\{10 \overline{1} 1\}$ pole figures (one dot per pole of each twin variant or parent crystal) corresponding to the initial Ti single crystal, and from VPSC simulations after $10 \%$ and $25 \%$ compressive strain along the [0001] direction. 


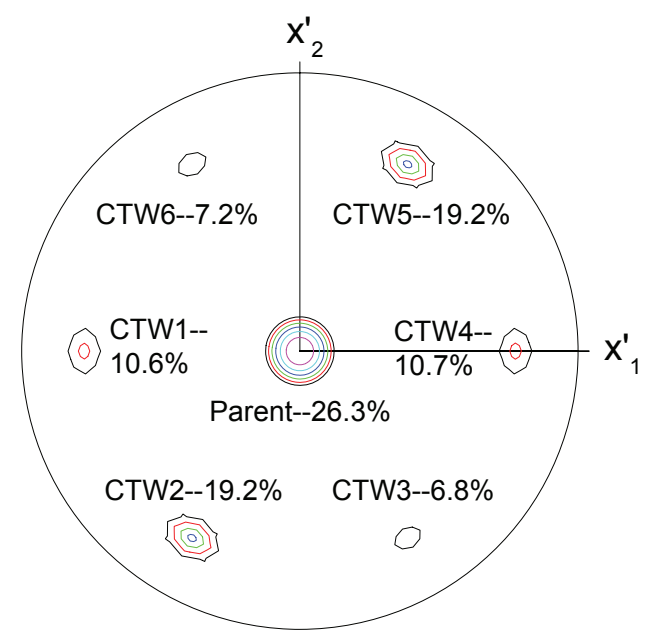

Figure 12: Basal (0001) pole figure, with compression axis $x_{3}$ parallel to the [0001] direction in the center, and two mutually perpendicular axes $x_{1}^{\prime}$ and $x_{2}^{\prime}$ at right and top, respectively, showing the predicted volume fractions of the different primary compressive twin variants and the remaining parent crystal, after $10 \%$ strain. 

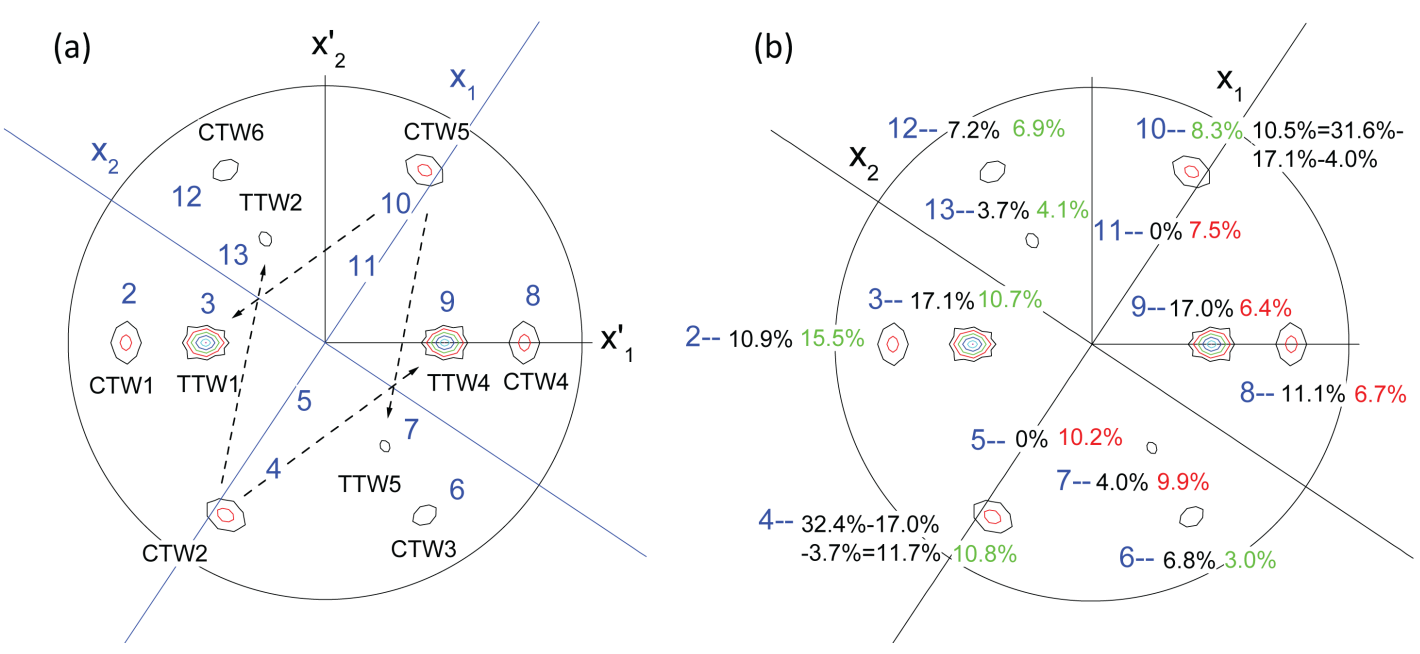

Figure 13: Basal (0001) pole figures after 25\% compressive strain along [0001]. Axes $x_{1}$ and $x_{2}$ correspond to the rotation around compression axis $x_{3}$ that give the best match between the predicted and measured intensities for regions 1 through 13 in Fig. 9(a). Predicted volume fractions for each primary and secondary twin variant are summarized in Table 5. The arrows in (a) indicate in which primary twin variant secondary twins were originated. (b) Predicted (in black) and measured (in color) volume fractions of the different labeled regions/twin variants. Measured volume fractions in green/red indicate reasonable/poor agreement with the corresponding predictions.

\begin{tabular}{|c|c|}
\hline Twin Type & Percentage \\
\hline CTW1 & $10.9 \%$ \\
\hline CTW2 & $32.4 \%$ \\
\hline CTW3 & $6.8 \%$ \\
\hline CTW4 & $11.1 \%$ \\
\hline CTW5 & $31.6 \%$ \\
\hline CTW6 & $7.2 \%$ \\
\hline
\end{tabular}

\begin{tabular}{|c|c|}
\hline Twin Type & Percentage \\
\hline TTW1 & $17.1 \%$ (from CTW5) \\
\hline TTW2 & $3.7 \%$ (from CTW2) \\
\hline TTW3 & $0 \%$ \\
\hline TTW4 & $17.0 \%$ (from CTW2) \\
\hline TTW5 & $4.0 \%$ (from CTW5) \\
\hline TTW6 & $0 \%$ \\
\hline
\end{tabular}

Table 5: VPSC prediction of volume fractions of different twin variant orientations for a Ti single crystal compressed along the [0001] direction. 

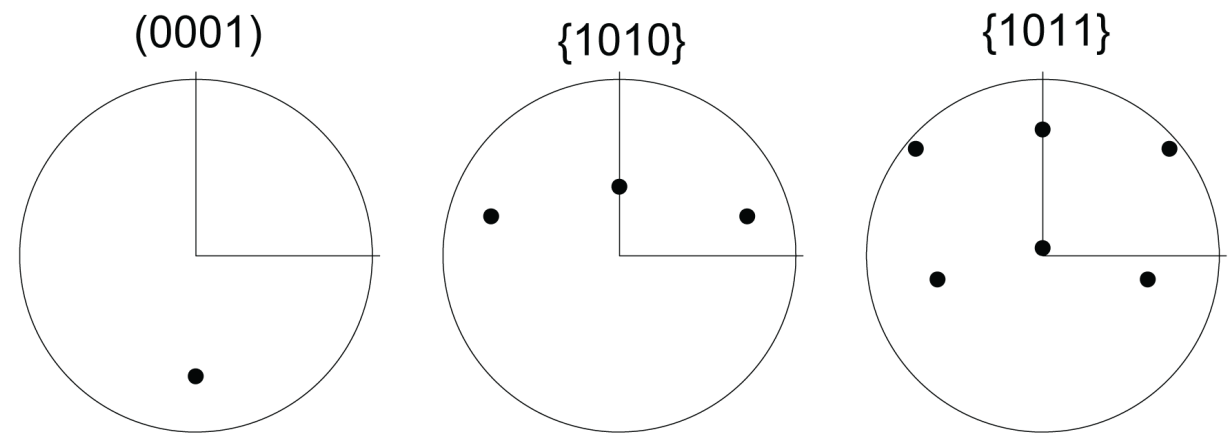

initial
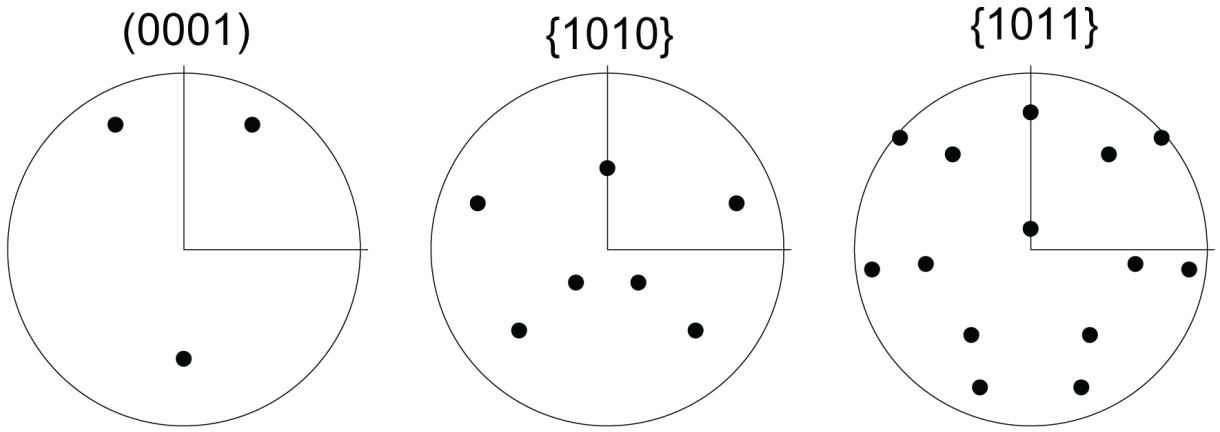

\section{$10 \%$}
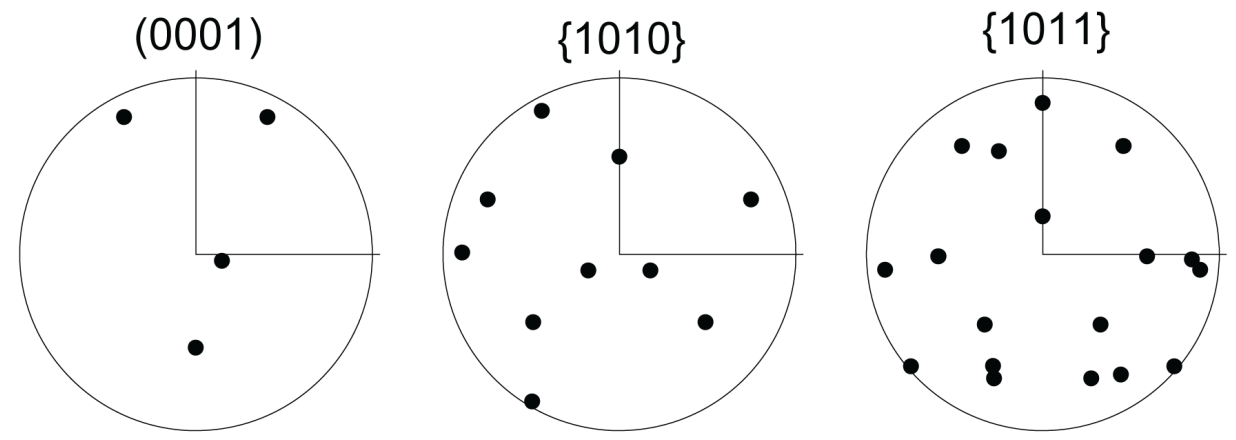

\section{$25 \%$}

Figure 14: Basal (0001), prismatic $\{10 \overline{1} 0\}$, and pyramidal $\{10 \overline{1} 1\}$ pole figures (one dot per pole of each twin variant or parent crystal) corresponding to the initial Ti single crystal, and from VPSC simulations after $10 \%$ and $25 \%$ compressive strain along the [1011] direction. 


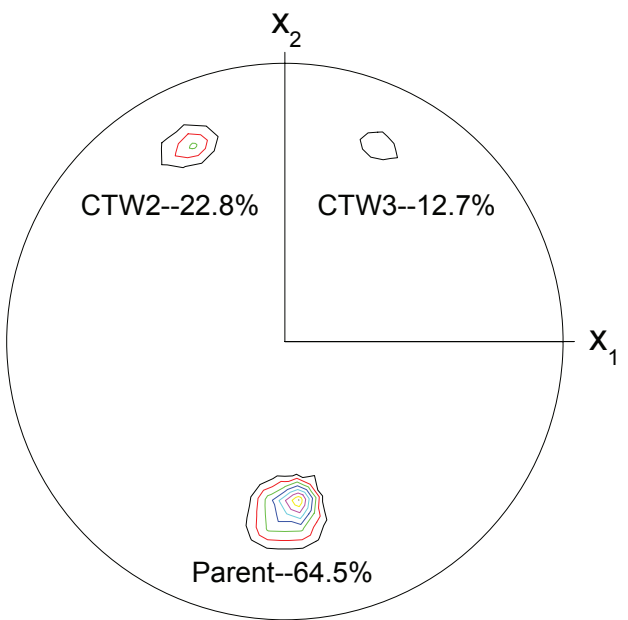

Figure 15: Basal (0001) pole figure showing the predicted volume fractions of the different primary compressive twin variants and the remaining parent crystal, after 10\% strain in the case of the Ti single crystal compressed along the $[10 \overline{1} 1]$ direction. 
(a)

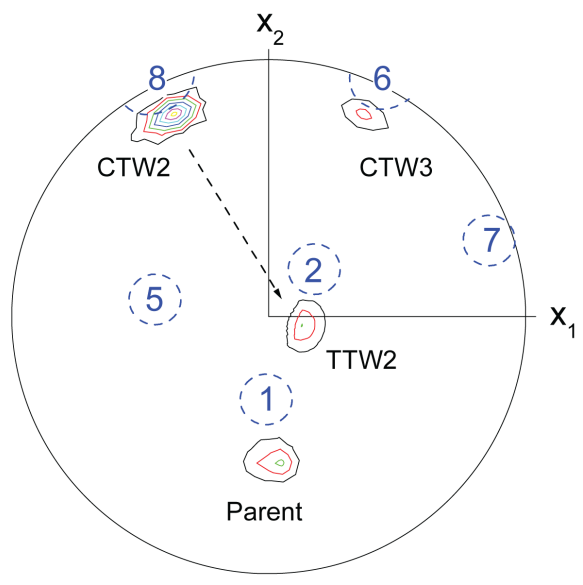

(b)

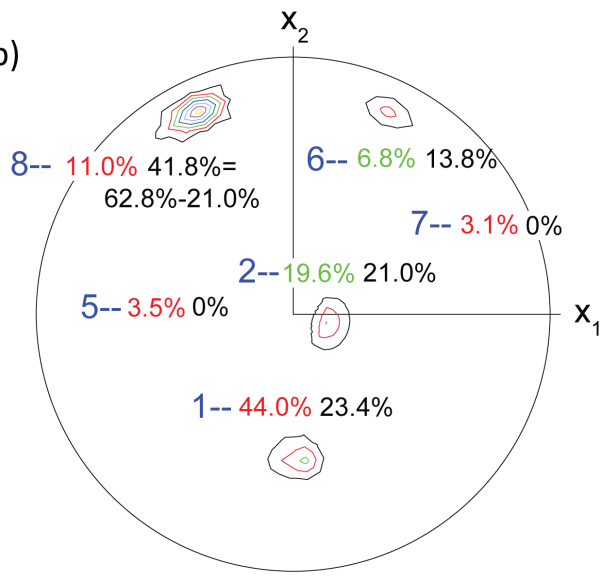

Figure 16: Basal (0001) pole figures after 25\% compressive strain along [1011]. Measured basal pole figure regions 1-2 and 5-8 from Fig. 9(b) are also shown. Predicted volume fractions for each primary and secondary twin variant are summarized in Table 6 . The arrow in (a) indicates in which primary twin variant secondary twins were originated. (b) Predicted (in black) and measured (in color) volume fractions of the different labeled regions/twin variants. Measured volume fractions in green/red indicate reasonable/poor agreement with the corresponding predictions.

\begin{tabular}{|c|c|}
\hline Twin Type & Percentage \\
\hline CTW1 & $0 \%$ \\
\hline CTW2 & $62.8 \%$ \\
\hline CTW3 & $13.8 \%$ \\
\hline CTW 4 & $0 \%$ \\
\hline CTW5 & $0 \%$ \\
\hline CTW6 & $0 \%$ \\
\hline
\end{tabular}

\begin{tabular}{|c|c|}
\hline Twin Type & Percentage \\
\hline TTW1 & $0 \%$ \\
\hline TTW2 & $21.0 \%$ (from CTW2) \\
\hline TTW3 & $0 \%$ \\
\hline TTW4 & $0 \%$ \\
\hline TTW5 & $0 \%$ \\
\hline TTW6 & $0 \%$ \\
\hline
\end{tabular}

Table 6: VPSC prediction of volume fractions of different twin variant orientations for a Ti single crystal compressed along the [1011] direction. 

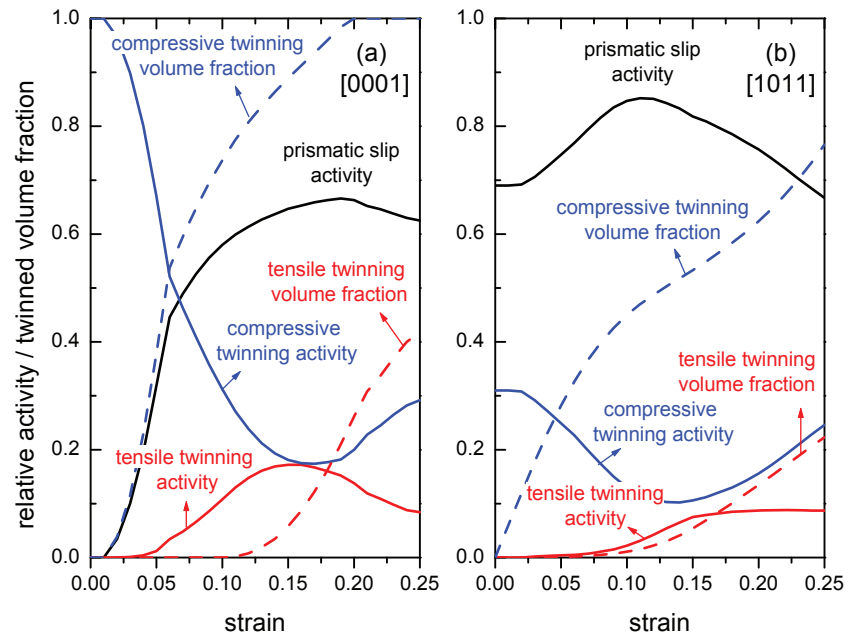

Figure 17: Relative activity of the slip and twinning modes and twinned volume fractions as a function of strain, for the [0001] and [101̄1] initial crystal orientations.
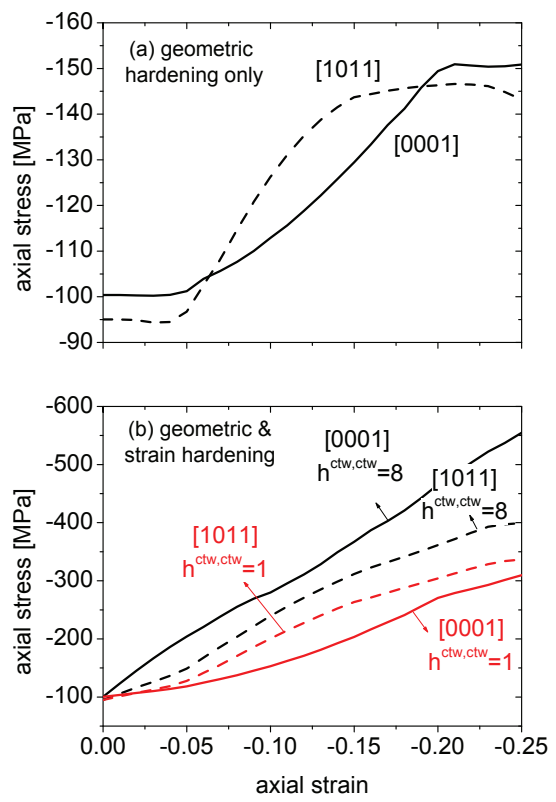

Figure 18: Stress-strain response for different strain hardening assumptions. (a) Case with no strain hardening, reflecting geometric hardening only. (b) Case with strain hardening, for no latent hardening (in red), and strong latent hardening between compressive twinning variants (in black). 
